\title{
A Numerical Study of Diffusion-Convection Equations
}

\author{
Imam Solekhudin \\ Department of Mathematics, Faculty of \\ Mathematics and Natural Sciences, \\ Universitas Gadjah Mada \\ Yogyakarta, Indonesia
}

\begin{abstract}
In this paper, problems involving timedependent diffusion-convection equation are studied. To study these problems, a numerical method is employed to solve the equation numerically. The method used in this research is a Laplace Transform Dual Reciprocity Method (LTDRM). Using this method, the time-dependent problem is transformed into a time-independent problem, which is easier to solve. A problem with the analytic solution is presented and solved using this method to analyze the accuracy of the method. Furthermore, a problem without an analytic solution is solved using the method. Results obtained are presented and discussed.
\end{abstract}

Keywords-diffusion-convection, LTDRM, numerical solutions

\section{INTRODUCTION}

Mathematical studies of diffusion-convection problems have been undertaken by numerous researchers. Such researchers are Codina [1], Barret and Morton [2], Herbin [3], and Morton [4]. Codina employed finite element methods for solving diffusion-convection reaction equation [1]. Galerkin methods were used by Barret and Morton to solve diffusion-convection problems [2]. While Herbin applied finite volume scheme for solving a diffusionconvection problem and estimated errors obtained from the method [3]. Morton used finite difference approximation to solve a diffusion-convection equation and analysed the stability of the method [4]. In these studies, some numerical methods have been employed to solve diffusion-convection problems.

In this paper, a different method is proposed to solve time-dependent diffusion-convection problems. The method is one of the meshless method known as the Laplace Transform Dual Reciprocity Method (LTDRM). Using this method, the governing equation, which is a time-dependent equation, is transformed into a time-independent equation. The time-independent equation is then solved using a dual reciprocity procedure. This method has more flexibility to solve problems compared to the Finite Element Method (FEM) or Finite Difference Method (FEM). This method does not require interpolation to obtain solutions at any points. This method has been successfully implemented to solve infiltration problems in homogeneous soil [5]. To implement the method, two diffusion-convection problems are considered, a problem with the analytic solution and a problem without an analytic solution. Numerical results obtained using LTRDM are then analysed.

\section{PROBLEM FORMULATION}

In this paper, problems involving time-dependent diffusion-convection equation without source terms are considered. The diffusion-convection equation in this study is

$$
\frac{\partial \nu}{\partial t}=\frac{\partial^{2} \nu}{\partial x^{2}}+\frac{\partial^{2} \nu}{\partial y^{2}}+V_{x} \frac{\partial \nu}{\partial x}+V_{y} \frac{\partial \nu}{\partial y},
$$

over a domain which can be presented as a union of a region $R$ and a simple closed curve $C$, where $t$ is the time, $v$ is a function of time and space $(v=v(x, y, t))$, and $V_{x}$ and $V_{y}$ are functions of $x$ and $y$.

In this study, a numerical method known as LTDRM is employed to solve the problems. The purpose of applying the method is to demonstrate the method to solve such problems and to investigate the accuracy of the method. These are useful as most of real life problems may not be solved analytically. To test the accuracy of the method, a problem with the analytic solution is presented. Furthermore, a problem without an analytic solution is then presented and solved numerically using the method. The two problems are as follows.

\section{A. Problem 1}

This problem is created by the author. In Problem 1, a diffusion-convection problem, with diffusion-convection equation

$$
\frac{\partial \nu}{\partial t}=\frac{\partial^{2} \nu}{\partial x^{2}}+\frac{\partial^{2} \nu}{\partial y^{2}}+2 \frac{\partial \nu}{\partial y}
$$

over a domain defined by

$$
D_{1}=\left\{(x, y) \in \mathbb{R}^{2}: 0 \leq x \leq \pi \text { and } 0 \leq y \leq \pi\right\}
$$

with initial condition

$$
\nu(0)=\sin x, \text { for }(x, y) \in D_{1},
$$

subject to boundary conditions

$$
\begin{aligned}
\nu & =0, \text { for } x=0 \text { and } 0 \leq y \leq \pi, \\
\frac{\partial \nu}{\partial n} & =0, \text { for } 0 \leq x \leq \pi \text { and } y=0, \\
\nu & =0, \text { for } x=\pi \text { and } 0 \leq y \leq \pi, \\
\frac{\partial \nu}{\partial n} & =0, \text { for } 0 \leq x \leq \pi \text { and } y=\pi,
\end{aligned}
$$

is considered. The analytic solution to Problem 1 is

$$
\nu=e^{-t} \sin x \text {. }
$$


As been stated earlier, this problem is used to test the accuracy of LTDRM.

\section{B. Problem 2}

In this problem, we consider a diffusion-convection equation

$$
\frac{\partial \nu}{\partial t}=\frac{\partial^{2} \nu}{\partial x^{2}}+\frac{\partial^{2} \nu}{\partial y^{2}}-\frac{\partial \nu}{\partial x}
$$

over a domain

$$
D_{2}=\left\{(x, y) \in \mathbb{R}^{2}: 0 \leq x \leq 1 \text { and } 0 \leq y \leq 1\right\} \text {, }
$$

with initial condition

$$
\nu(0)=0, \text { for }(x, y) \in D_{2},
$$

Subject to boundary conditions

$$
\begin{aligned}
\nu & =1, \text { for } x=0 \text { and } 0 \leq y \leq 1, \\
\frac{\partial \nu}{\partial n} & =0, \text { for } 0 \leq x \leq 1 \text { and } y=0, \\
\frac{\partial \nu}{\partial n} & =0, \text { for } x=1 \text { and } 0 \leq y \leq 1, \\
\frac{\partial \nu}{\partial n} & =0, \text { for } 0 \leq x \leq 1 \text { and } y=1 .
\end{aligned}
$$

In real-life situations, this initial boundary equation describes the movement of particles with the source at line $x$ $=0$. At $t=0$, there are no such particles in the interior of the domain. The particles diffuse with a diffusion coefficient of 1. There is a "stream" flowing in the direction of the positive $x$-axis, with a velocity of 0.1 . In this problem, the analytic solution of the problem is not given.

\section{METHOD OF SOLUTION USING LTDRM}

In this section, the method of solution is presented briefly. Readers may refer to [6] and [7] for detail of the method. In [6] and [7] the method was applied to solve problems without convection terms. In this study, the method is applied to solve problems with convection terms. To apply the method, a Laplace transform defined as

$$
\nu^{*}(x, y, s)=\int_{0}^{\infty} e^{-s t} \nu(x, y, t) d t
$$

with $v(x, y, 0)=v_{0}$.

Using Laplace transform (17), Equation (1) may be transformed to

$$
s \nu^{*}-\nu_{0}=\frac{\partial^{2} \nu^{*}}{\partial x^{2}}+\frac{\partial^{2} \nu^{*}}{\partial y^{2}}+V_{x} \frac{\partial \nu^{*}}{\partial x}+V_{y} \frac{\partial \nu^{*}}{\partial y} .
$$

An integral equation resulted from Equation (18) is

$$
\begin{array}{r}
\lambda(\xi, \eta) \nu^{*}(\xi, \eta, s)=\int_{C}\left[\nu^{*}(x, y, s) \frac{\partial}{\partial n}(\Theta(x, y ; \xi, \eta))\right. \\
\left.-\Theta(x, y ; \xi, \eta) \frac{\partial}{\partial n}\left(\nu^{*}(x, y, s)\right)\right] d s \\
+\iint_{R} \Theta(x, y ; \xi, \eta)\left[s \nu^{*}(x, y, s)\right. \\
-\left(V_{x}(x, y) \frac{\partial}{\partial x}\left(\nu^{*}(x, y, s)\right)\right. \\
\left.\left.+V_{y}(x, y) \frac{\partial}{\partial y}\left(\nu^{*}(x, y, s)\right)+\nu_{0}\right)\right] d x d y
\end{array}
$$

where

$$
\Theta(x, y ; \xi, \eta)=\frac{1}{2 \pi} \ln \sqrt{(x-\xi)^{2}+(y-\eta)^{2}}
$$

is the fundamental solution of two dimensional Laplace equation, and

$$
\lambda(\xi, \eta)=\left\{\begin{array}{ll}
1, & (\xi, \eta) \in R \\
\frac{1}{2}, & (\xi, \eta) \text { on the smooth part of } C
\end{array} .\right.
$$

Boundary integral equation (19) may be recast into a system of linear algebraic equations

$$
\begin{aligned}
& \lambda^{(n)} \nu^{*(n)}= \\
& \sum_{k=1}^{N}\left[\mathcal{F}_{2}^{(k)}\left(a^{(n)}, b^{(n)}\right) \nu^{*(k)}-\mathcal{F}_{1}^{(k)}\left(a^{(n)}, b^{(n)}\right) \nu_{n}^{*(k)}\right] \\
& +\sum_{j=1}^{N+L}\left\{\left[s \mu^{(n j)}-\mu_{x}^{(n j)} V_{x}^{(j)}-\mu_{y}^{(n j)} V_{y}^{(j)}\right] \nu^{*(j)}-\mu^{(n j)} \nu_{0}\right\}, \\
& n=1,2, \cdots, N+L,
\end{aligned}
$$

where

$$
\begin{aligned}
& \lambda^{(n)}=\lambda\left(a^{(n)}, b^{(n)}\right), \nu^{*(n)}=\nu^{*}\left(a^{(n)}, b^{(n)}\right), \\
& V_{x}^{(j)}=V_{x}\left(a^{(j)}, b^{(j)}\right), V_{y}^{(j)}=V_{y}\left(a^{(j)}, b^{(j)}\right), \\
& \nu_{n}^{*(k)}=\left.\frac{\partial \nu^{*}}{\partial n}\right|_{(x, y)=\left(a^{(k)}, b^{(k)}\right)}, \quad \mu^{(n j)}=\sum_{m=1}^{N+L} \Psi^{(n m)} \omega^{(m j)} \\
& \mathcal{F}_{1}^{(k)}\left(a^{(n)}, b^{(n)}\right)=\int_{C^{(k)}} \Theta\left(x, y ; a^{(n)}, b^{(n)}\right) d s, \\
& \mathcal{F}_{2}^{(k)}\left(a^{(n)}, b^{(n)}\right)=\int_{C^{(k)}} \frac{\partial}{\partial n}\left[\Theta\left(x, y ; a^{(n)}, b^{(n)}\right)\right] d s, \\
& \mu_{x}^{(n j)}=\sum_{l=1}^{N+L}\left[\mu^{(n l)}\left(\sum_{m=1}^{N+L} \rho_{x}^{(l m)} \omega^{(m j)}\right)\right], \\
& \text { and } \mu_{y}^{(n j)}=\sum_{l=1}^{N+L}\left[\mu^{(n l)}\left(\sum_{m=1}^{N+L} \rho_{y}^{(l m)} \omega^{(m j)}\right)\right] .
\end{aligned}
$$


Here

$$
\rho_{x}=\frac{\partial \rho}{\partial x}, \quad \rho_{y}=\frac{\partial \rho}{\partial y},
$$

where $\rho$ is defined as

$$
\rho(x, y ; a, b)=1+r^{2}(x, y ; a, b)+r^{3}(x, y ; a, b),
$$

with

$$
r(x, y ; a, b)=\sqrt{(x-a)^{2}+(y-b)^{2}} .
$$

Notations $\rho^{(l j)}, \rho_{x}^{(l j)}, \rho_{y}^{(l j)}, \omega^{(m k)}$, and $\Psi^{(n m)}$ are as follows.

$$
\begin{gathered}
\rho^{(l j)}=\rho\left(a^{(l)}, b^{(l)} ; a^{(j)}, b^{(j)}\right), \quad \rho_{x}^{(l j)}=\rho_{x}\left(a^{(l)}, b^{(l)} ; a^{(j)}, b^{(j)}\right), \\
\rho_{y}^{(l j)}=\rho_{y}\left(a^{(l)}, b^{(l)} ; a^{(j)}, b^{(j)}\right), \\
\sum_{k=1}^{N+L} \omega^{(m k)} \rho^{(k l)}=\left\{\begin{array}{ll}
1, & \text { if } k=m \\
0, & \text { if } k \neq m
\end{array},\right.
\end{gathered}
$$

and

$$
\begin{aligned}
\Psi^{(n m)}= & \Psi\left(a^{(n)}, b^{(n)} ; a^{(m)}, b^{(m)}\right) \\
=\quad \lambda\left(a^{(n)}, b^{(n)}\right) \chi\left(a^{(n)}, b^{(n)} ; a^{(m)}, b^{(m)}\right) & \\
+\int_{C} & {\left[\Phi\left(x, y ; a^{(n)}, b^{(n)}\right) \frac{\partial}{\partial n}\left[\chi\left(x, y ; a^{(m)}, b^{(m)}\right)\right]\right.} \\
& \left.-\chi\left(x, y ; a^{(m)}, b^{(m)}\right) \frac{\partial}{\partial n}\left[\Phi\left(x, y ; a^{(n)}, b^{(n)}\right)\right]\right] d s .
\end{aligned}
$$

Function $\chi$ is defined as

$$
\begin{aligned}
\chi(x, y ; a, b)= & \frac{1}{4} r^{2}(x, y ; a, b)+\frac{1}{16} r^{4}(x, y ; a, b) \\
& +\frac{1}{25} r^{5}(x, y ; a, b) .
\end{aligned}
$$

Numbers $N$ and $M$ are the number of boundary elements and interior collocation points, respectively. Points $\left(a^{(k)}, a^{(k)}\right)$, $k=1,2, \ldots, N+L$, are the collocation points.

Numerical solutions of $v^{*}$ and $v_{n}{ }^{*}$ are obtained by solving System of linear algebraic equations (20). Using these numerical solutions, numerical solutions of $v^{*}$ at any point may be obtained. From $v^{*}$ obtained, numerical value of $v$ may be computed using an inverse Laplace transform known as Stehfest formula [6]

$$
\nu(x, y, t)=\frac{\ln 2}{t} \sum_{n=1}^{2 P} K_{n} \nu^{*}\left(x, y, s_{n}\right),
$$

where

$$
\begin{aligned}
s_{n} & =n \frac{\ln 2}{t}, \\
K_{n} & =(-1)^{(n+P)} \sum_{m=(n+1) / 2}^{\min (n, P)} \frac{m^{P}(2 m) !}{(P-m) ! m !(m-1) !(n-m) !(2 m-n) !},
\end{aligned}
$$

and $P$ is a positive integer.

\section{RESULTS AND DISCUSSION}

The method of solution is tested to obtain numerical solutions to Problem 1 and Problem 2. In Problem 1, the analytic solution to the problem is given. This solution is used to test the accuracy of the LTDRM. To apply the LTDRM, the boundary of the problem is descritized into a number of elements, and interior collocation points are chosen.

To implement the method for solving Problem 1 numerically, the boundary is discretized into 100 elements, and the number of interior points chosen is 225. These numbers are chosen after some computational experiment, so that numerical solutions are accurate to three decimal numbers. Some of numerical results obtained using the LTDRM are presented in Fig. 1. In the figure, numerical results are compared to the corresponding analytical results.

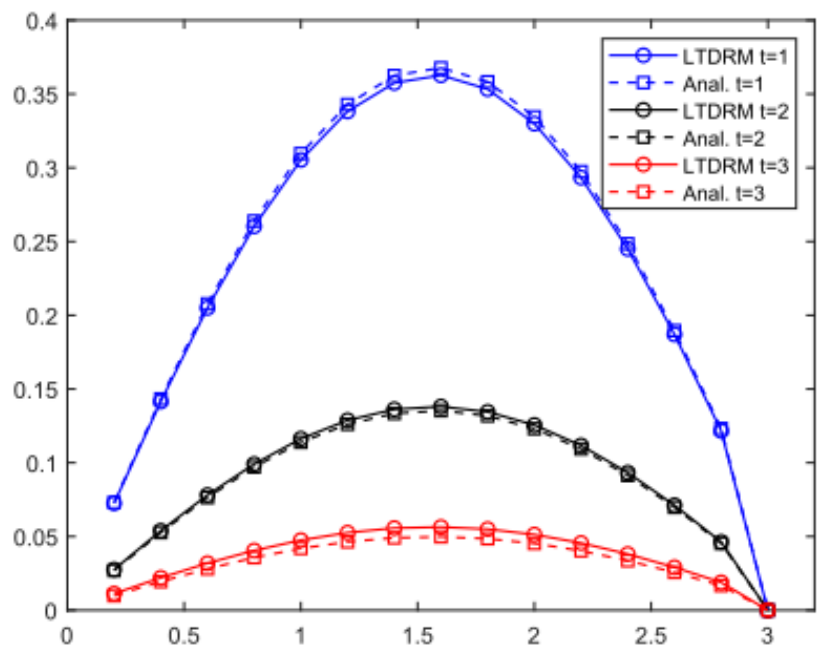

Fig. 1. Numerical and corresponding analytic solutions at selected points along line $y=0.2$.

From the figures, it can be seen that numerical solutions are in good accuracy with analytic solutions. It seems that absolute errors of the numerical solutions are less than 0.01 . It can be observed that the smallest error is at $x=0$ and $x=1$, while the highest error is at $x=0.5$. However, the error values are still tolerable for the set of segments and interior collocation points. Hence, the number of segments is applied to obtain numerical results of Problem 2.

To implement the LTDRM for solving Problem 2, 100 elements and 81 interior points are chosen. The number of elements is the same as that in the implementation of the LTDRM for solving Problem 1, as it has been shown that this number of elements results in accurate numerical solutions to Problem 1. The number of interior points is smaller than that in the implementation of the LTDRM for Problem 1, to reduce the computational time. It has been shown that decreasing the number of interior points does not contribute to the accuracy significantly [6]. Some of the results obtained are presented in Fig. 2 and Fig. 3. 


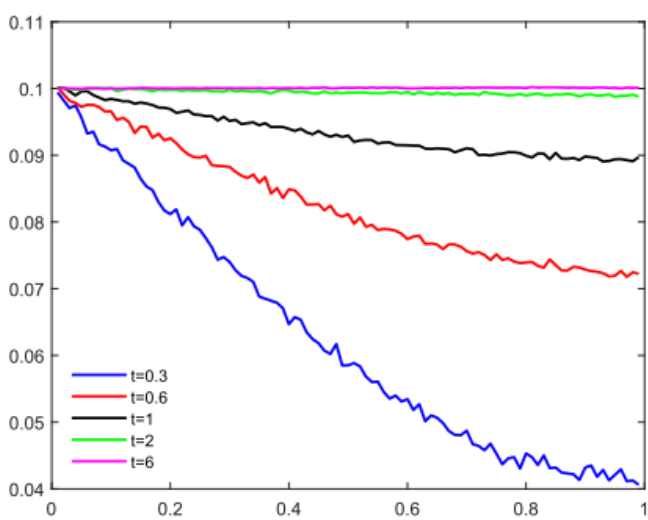

(a)

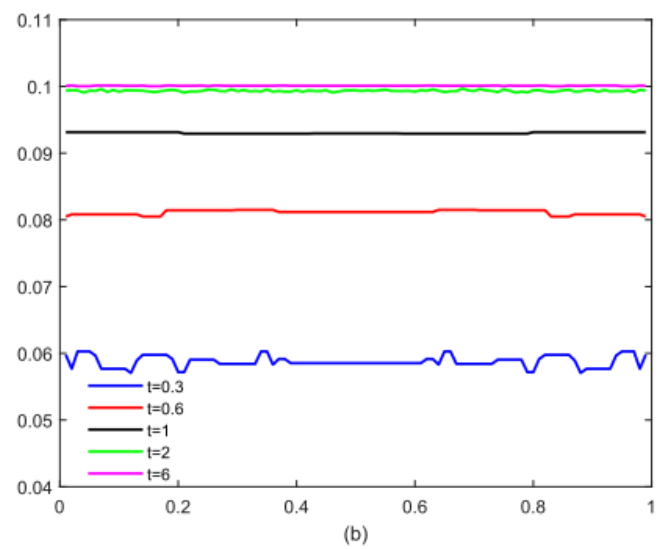

Fig. 2. Graphs of $v$ at different values of $t$. (a) At $y=0.5$ for $0<x<1$. (b) At $x=0.5$ for $0<\mathrm{y}<1$.

Fig. 2 shows graphs of $v$ at $t=0.3, t=0.6, t=1, t=2$, and $t=6$. Specifically, Fig. 2(a) presents graphs of $v$ along line $y=0.5$, and Fig. 2(b) display graphs of $v$ along line $x=$ 0.5 . It can be seen that value of $v$ is about 0.1 at locations near $x=0$ for any value of $t$. This is expected as $v=1$ at $x=$ 0 . There are drastic changes in the values of $v$ from $t=0.3$ to $t=0.6$, especially at $x=1$. At $x=1$, significant increases in $v$ are observed from $t=0.6$ to $t=1$. The increase in $v$ is still observed until $t=2$. After $t=2$, it seems that values of $v$ have reached their steady state values. Distribution of $v$ at different values of $t$ are shown in the surface plots in Fig. 3.
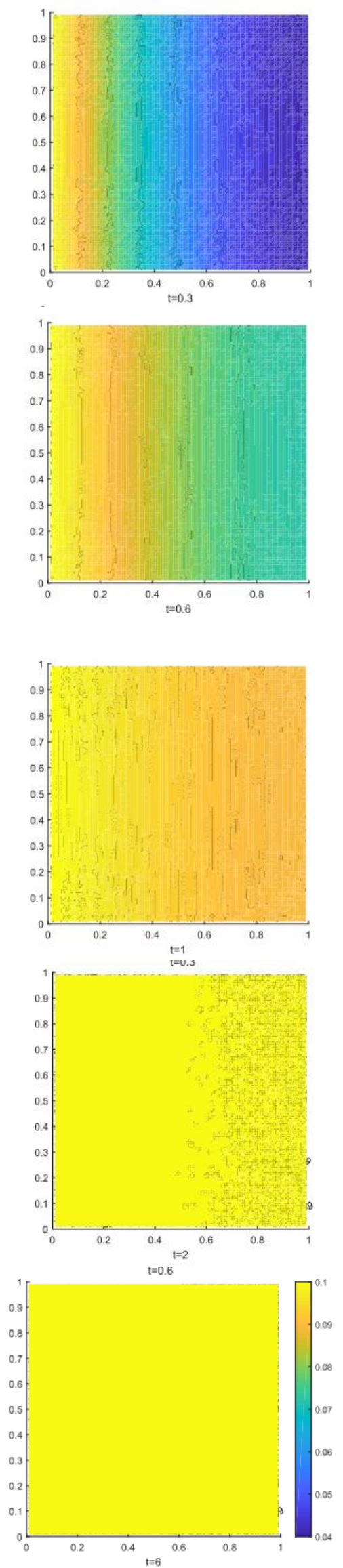

Fig. 3. Surface plots of $v$ over the domain at selected values of $t$. 
Fig. 3 shows the dynamics of changes in the value of $v$ over the domain. As can be seen in Fig. 3, changes in $v$ are observed until $t=2$. After $t=2$, there are no much changes in $v$ occur on the domain. Moreover, at $t=6$, values of $v$ are about constant over the domain. These results show that at $t$ $=2$, the distribution of $v$ over the domain is approaching that at steady state condition. Furthermore, it seems that steady state conditions has been reached at $t=6$.

\section{CONCLUSION}

Diffusion-convection problems has been considered in this study. A Laplace Transform Dual Reciprocity Method (LTDRM) has been formulated and implemented to solve the problems numerically. Results show that numerical results obtained using this method are in good accuracy with the corresponding analytical solutions. Furthermore, for the problem with unknown analytical solution, dynamics of changes of numerical solutions are presented. Steady state conditions have been achieved at $t=6$.

\section{REFERENCES}

[1] Codina, R., "Comparison of some finite element methods for solving the diffusion-convection-reaction equation," Computer Methods in Applied Mechanics and Engineering, vol. 156, pp. 185 - 210 , 1998.

[2] Barret, J.W. and Morton, K.W., "Approximate symmetrization and Petrov-Galerkin methods for diffusion-convection problems," Computer Methods in Applied Mechanics and Engineering, vol. 45, pp. 97 - 122, 1984.

[3] Herbin, R., "An error estimate for a finite volume scheme for a diffusion-convection problem on a triangular mesh," Numerical Methods for Partial Differential Equations, vol. 11, pp. 165 - 170, 1995.

[4] Morton, K.W., "Stability of finite difference approximations to a diffusion-convection equation," International Journal for Numerical Methods in Engineering, vol. 15, pp. 677 - 685,1980

[5] Solekhudin, I. and Ang K.C., "A Laplace Transform DRBEM with a Predictor-Corrector Scheme for Time Dependent Infiltration from Periodic Channels with Root Water Uptake," Engineering Analysis with Boundary Elements, vol. 50, pp. 141 - 145, 2015

[6] Solekhudin, I., "Dual reciprocity boundary element methods for water infiltration problems in irrigation," $\mathrm{PhD}$ Thesis, NIE-NTU, Singapore, 2013.

[7] Lobo, M., "Boundary element methods for a class of infiltration problems" PhD Thesis, The University of Adelaide, Australia, 2008. 